\title{
Primary localized cutaneous amyloidosis with lichen and poikiloderma- like lesions and an excellent response to systemic acitretin ${ }^{*}$
}

\author{
Han $\mathrm{MA}^{1}$ \\ Guoxing $\mathrm{Zhu}^{1}$ \\ Chun $\mathrm{Lu}^{1}$
}

\author{
Xiangyang $\mathrm{Su}^{1}$ \\ Songchao Yin ${ }^{1}$ \\ Wei Lai ${ }^{1}$
}

DOI: http://dx.doi.org/10.1590/abd1806-4841.20164316

\begin{abstract}
Primary localized cutaneous amyloidosis is a skin-limited amyloidosis that does not involve internal organs. It is clinically subclassified into 3 general categories and some rare variants. However, there is considerable overlap within the classification. Though there are a variety of therapeutic measures, the treatment is often unsatisfactory, particularly when the disease is severe and extensive. We describe a rare case of primary localized cutaneous amyloidosis with lichen and poikiloderma-like lesions that showed an excellent response to systemic acitretin.
\end{abstract}

Keywords: Acitretin; Amyloidosis; Lichens

\section{INTRODUCTION}

Primary localized cutaneous amyloidosis (PLCA) is characterized by the limited deposition of amyloid in the skin, without deposition in any internal organs. ${ }^{1}$ There are a variety of clinical phenotypes and treatment is often difficult, particularly when the disease is severe and extensive. In this study, we describe a rare case of PLCA with lichen and poikiloderma-like lesions and an excellent response to systemic acitretin.

\section{CASE REPORT}

A 22-year-old woman had endured multiple, mildly pruritic, red-purple skin lesions on her face, external ear, neck and upper back for twenty years (Figure 1) ${ }^{1-3}$ The eruptions initially developed as papules on her face and spread gradually to the other locations. Some lesions on the forehead and bilateral parts of the malar region and cheeks (is this a repetition of the same idea?) coalesced into plaques, while others on the upper back partly subsided spontaneously, leaving hypopigmented macules. Her medical history was unremarkable and there were no reports of a similar condition in her family members. Physical examination revealed symmetric, multiple, red-purple, hyperkeratotic papules and plaques on the face, external ear and neck. The papules were intermingled with hypopigmented macules and telangiectatica, producing a reticulated appearance on the upper back. Laboratory examination showed no abnormality in routine tests, including blood count, urinalysis, liver and kidney function test, muscle enzymes and serum uric acid lev- el. Serum and urine immunofixation returned negative results. No cardiac abnormalities were noted upon echocardiography. A skin biopsy specimen revealed hyperkeratosis, focal hypergranulosis, irregular acanthosis, liquefaction degeneration of the basal layer, and aggregations of amorphous eosinophilic material and pigment incontinence in the papillary dermis (Figure 2A). The eosinophilic material stained positively with Congo red (Figure 2B). Based on the clinical manifestations and histopathological features, the patient was diagnosed with PLCA and treated with systemic acitretin at an initial dose of $0.5 \mathrm{mg} / \mathrm{kg} / \mathrm{d}(30 \mathrm{mg} / \mathrm{d})$. The patient experienced clear relief from pruritus and saw some improvement in the papules and plaques 6 months later, with mild mucocutaneous side effects (Figure 1). ${ }^{1-3}$ Furthermore, 18 months later, (later was missing but presumably that is what was meant?) the case showed a remarkably good response to the medicine (Figure 1) $\cdot^{1-3}$

\section{DISCUSSION}

Amyloidosis refers to a heterogeneous group of disorders characterized by extracellular deposition of proteinaceous fibrillar materials (termed amyloid) in various tissues and organs. ${ }^{2}$ At present, there are 30 structurally unrelated proteins that are known to cause amyloidosis. ${ }^{3}$ PLCA is a skin-limited amyloidosis that does not involve internal organs. Histologically, it is characterized by the deposition of amyloid in the superficial dermis. Clinically, it is subclassified into 3 general categories: lichen (papular); macular

Received on 19.12.2014.

Approved by the Advisory Board and accepted for publication on 26.01.2015.

* Work performed at the Third Affiliated Hospital, Sun Yat-sen University - Guangdong, China.

Financial Support: None.

Conflict of Interest: None.

1 Third Affiliated Hospital, Sun Yat-sen University - Guangdong, China.

(C)2016 by Anais Brasileiros de Dermatologia 

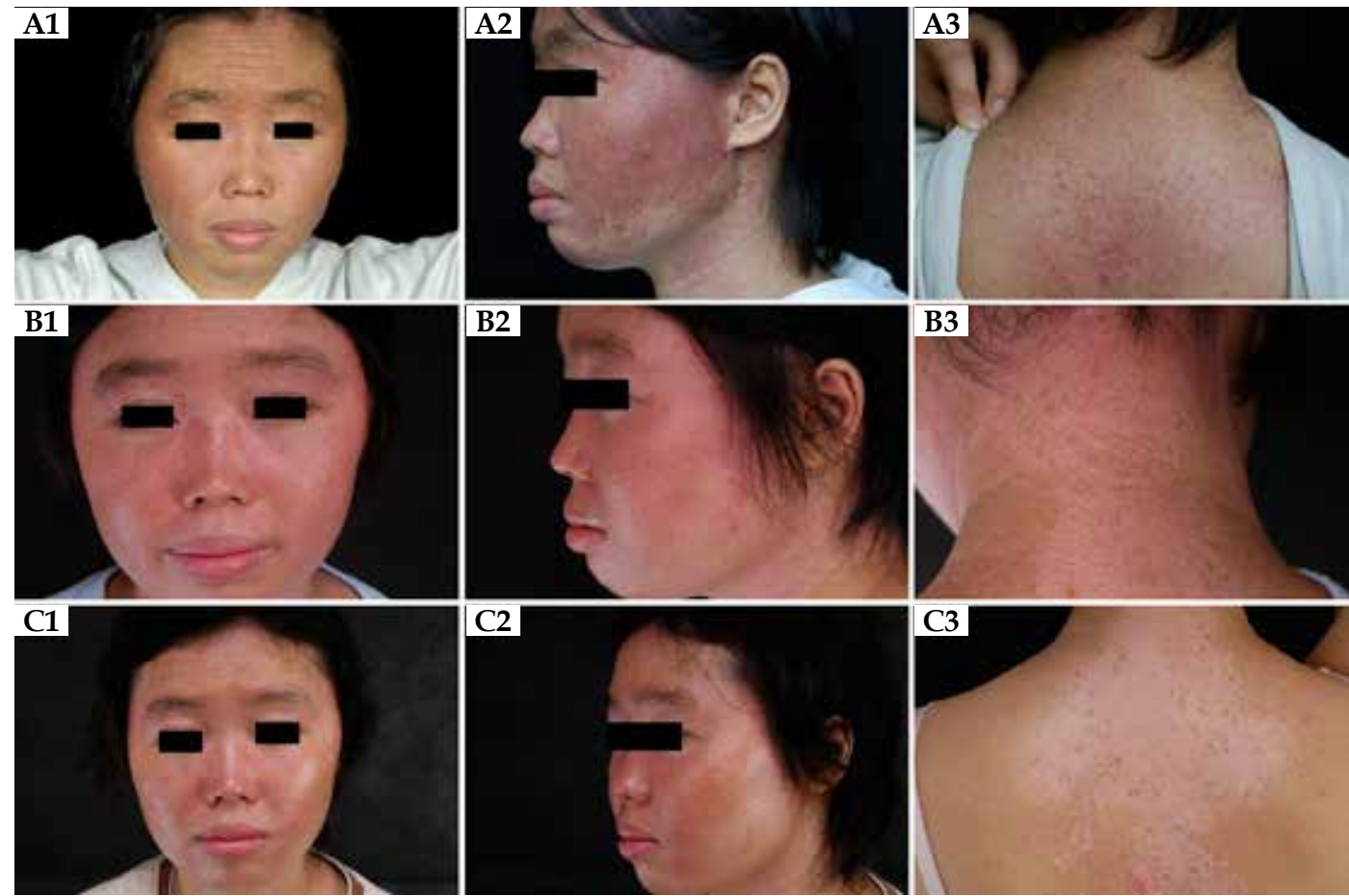

Figure 1:

Skin lesions on the face $(\mathrm{A} 1, \mathrm{~A} 2)$ and upper back (A3). 6 months (B1-3) and 18 months (C1-3) after treatment with systemic acitretin
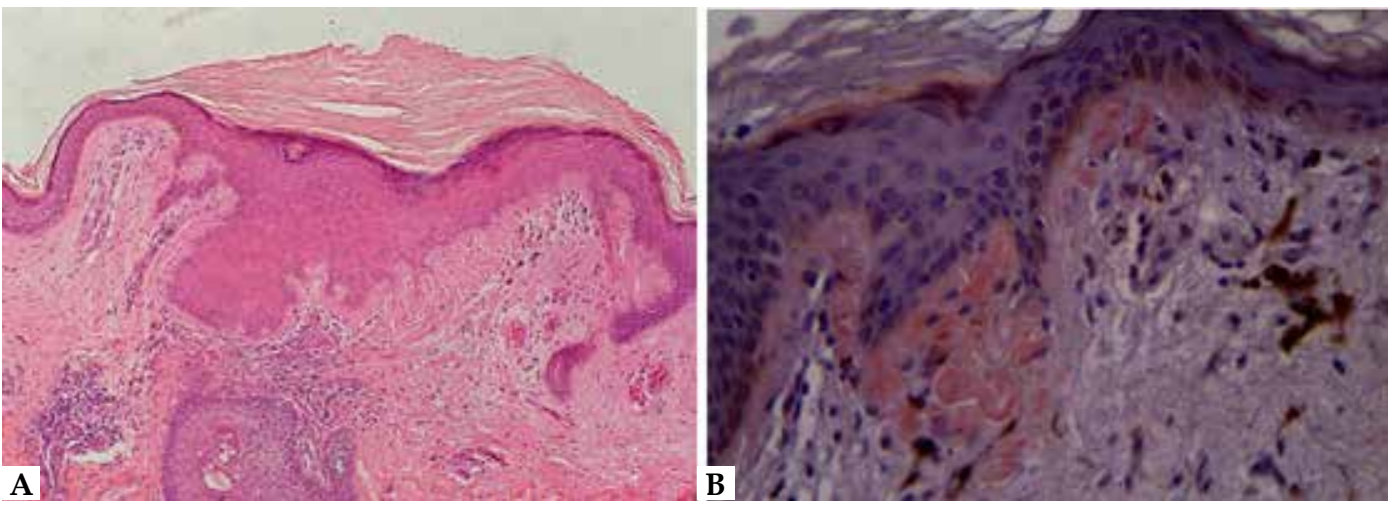

Figure 2:

The histological findings. (A) Hyperkeratosis, liquefaction degeneration of the basal layer, aggregations of amorphous eosinophilic material and pigment incontinence in the papillary dermis $(\mathrm{H} \&$ E, 100×). (B) The eosinophilic material stained positively (Congo red stain, $400 \times$ )

(the most common type); and nodular amyloid (the rarest). ${ }^{4}$ Rare variants include the poikiloderma-like, bullous and vitiliginous types, while anosacral forms have also been reported. However, the classification involves considerable overlap, both within the subtypes of PLCA and with systemic amyloidosis. ${ }^{5}$ This protean clinical manifestation also appears in patients with lichen lesions on the face, external ear, neck and poikiloderma-like lesions on the upper back.

Initial amyloid formation in PLCA is thought to be related to keratinocyte apoptosis. But the general mechanism of amyloid fibril formation is not fully understood. Koji Ono et al. proposed that the possible mechanism for promoting amyloid fibril formation in PLCA may be summarized in 6 steps. Thus, galectin-7 (Gal-7) may be an amyloid precursor protein, while actin and cytokeratins may be involved in modulating the aggregation of amyloidogenic peptides. ${ }^{6}$
Treating PLCA is difficult and numerous methods have been tried with variable success. Systemic etretinate or acitretin appeared to be helpful in some cases, just seen in this patient. The possible mechanisms were proposed as follows: First, retinoids have anti-inflammatory and anti-proliferative actions that inhibit epidermal acanthosis and further inflammatory injury to basal keratinocytes. They are known to promote keratinocyte differentiation and can prevent amyloidogenesis by correcting a keratin 5:14 imbalance. Second, they can regulate levels of several heat-shock proteins that may prevent aberrant folding of keratin into amyloids. Third, retinoids can also reduce apolipoprotein E secretion, a protein found together with amyloids that is considered crucial in promoting its formation. ${ }^{7}$ As Gal-7 is an important amyloid precursor protein in PLCA, further studies are necessary to investigate the association between Gal-7 and retinoids.] 


\section{REFERENCES}

1. Norisugi 0, Yamakoshi T, Shimizu T. Successful treatment of lichen amyloidosis using a CO2 surgical laser. Dermatol Ther. 2014;27:71-3.

2. Dember LM. Amyloidosis-associated kidney disease. J Am Soc Nephrol. 2006;17:3458-71.

3. Sipe JD, Benson MD, Buxbaum JN, Ikeda S, Merlini G, Saraiva MJ, et al. Amyloid fibril protein nomenclature: 2012 recommendations from the Nomenclature Committee of the International Society of Amyloidosis. Amyloid. 2012;19:167-70.

4. Brinster N, Calonje E. The amyloidoses. In: Calonje JE, Brenn T, eds. McKee's Pathology of the Skin. 4th ed. Amsterdam: Elsevier Saunders; 2011. p.532-543.

5. Chandran NS, Goh BK, Lee SS, Goh CL. Case of primary localized cutaneous amyloidosis with protean clinical manifestations: lichen, poikiloderma-like, dyschromic and bullous variants. J Dermatol. 2011;38:1066-71.

6. Chandran NS, Goh BK, Lee SS, Goh CL. In vitro Amyloidogenic Peptides of Galectin-7: Possible Mechanism of Amyloidogenesis of Primary Localized Cutaneous Amyloidosis. J Dermatol. 2011;38:1066-71.

7. Choi JY, Sippe J, Lee S. Acitretin for lichen amyloidosus. Australas J Dermatol. 2008;49:109-13.
MAILING ADDRESS:

Xiangyang $S u$

Department of Dermatology

the Third Affiliated Hospital

Sun Yat-sen University, 600 Tianhe Road

Guangzhou

Guangdong, 510630, China

E-mail: sh3355@vip.163.com

How to cite this article: MA H, Su X, Zhu G, Yin S, Lu C, Lai W. Primary localized cutaneous amyloidosis with lichen and poikiloderma-like lesions and an excellent response to systemic acitretin. An Bras Dermatol. 2016;91(5):661-3. 